\title{
PRELIMINARY REPORT OF CATARACT EXTRACTION BY FREEZING*
}

\author{
BY \\ JOSEPH CONWAY \\ Regional Eye Centre, Whipps Cross Hospital, London
}

THIS paper is a preliminary report of a clinical trial to assess the value of cataract extraction by freezing.

Thirty patients underwent cataract extraction, the operation being performed by the author using a probe at a low temperature.

Recent developments in the science and techniques of very low temperatures (cryogenics) have suggested important potential applications in the field of ophthalmic surgery.

Investigations into the effects of low temperatures have been carried out over many years. In 1665 Robert Boyle published a series of essays concerning experiments and observations on the effects of cold. This was 49 years before Fahrenheit invented the first accurate thermometer. Low-temperature techniques have been used in corneal preservation (Billingham, 1957; Ridge, 1957), but it is only recently that these techniques have been used in cataract surgery.

Krwawicz $(1961$; 1963) described a method of intracapsular cataract extraction using an instrument (cryoextractor) which was placed in a cylinder of ethyl alcohol and cooled by a mixture of solid carbon dioxide and methyl alcohol at a temperature of $-79^{\circ} \mathrm{C}$. The method reported by Davies (1965) has been currently in use at Whipps Cross Hospital (using industrial methylated spirit B.P. instead of methyl alcohol).

The cryoextractor described by Krwawicz has certain disadvantages. When removed from the cooling mixture to room temperature it rapidly heats up, so limiting the time available for its use in cataract extraction. Also the sterilized ethyl alcohol, in final contact with which the instrument is cooled, may inadvertently be transferred into the eye. The insulation of the instrument is such that the cornea or iris may accidentally be touched and adhere to the cold metal above the tip with resulting possible damage.

In an attempt to overcome the above disadvantages a simple instrument has been produced and cooled with liquid nitrogen. The instrument consists of a pencil-

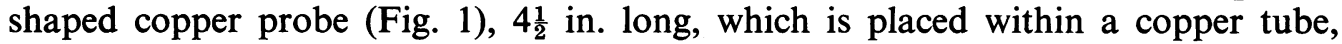
sterilized, and then suspended in a vacuum flask of liquid nitrogen (Fig. 2).

To ensure asepsis the probe itself does not come into contact with the liquid nitrogen. The latter is an ideal refrigerant with a temperature of $-196^{\circ} \mathrm{C}$., it vaporizes without leaving any residue, and the gas is inert, colourless, odourless,

\footnotetext{
* Received for publication August 17, 1964.
} 


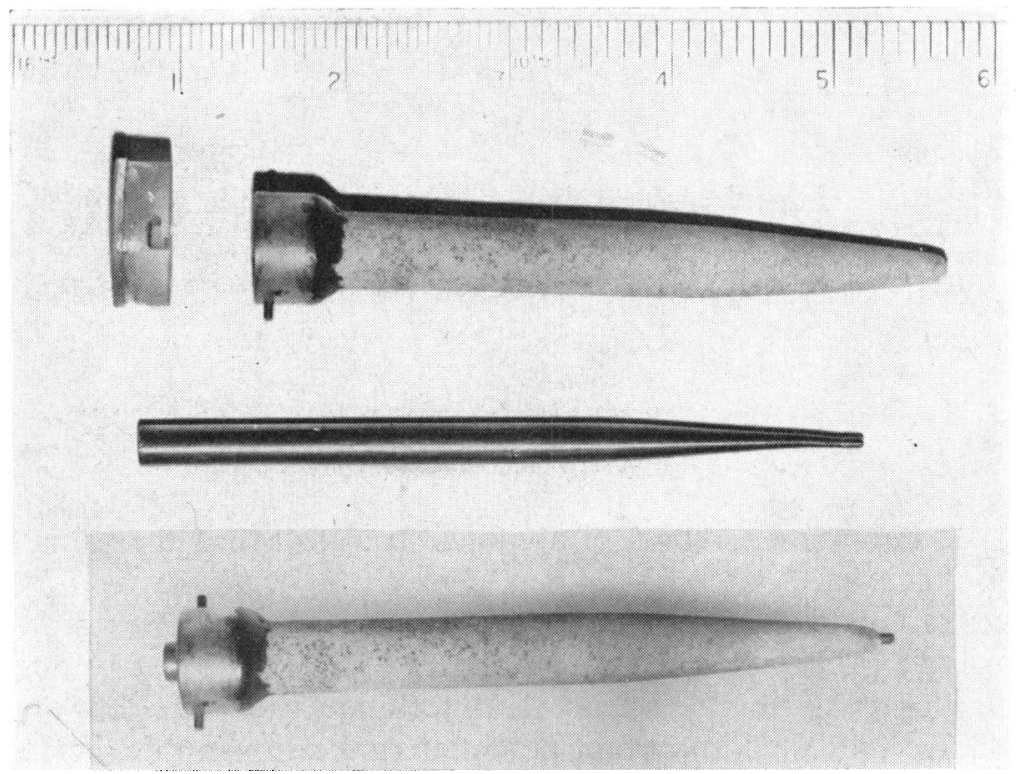

Fig. 1.-Top, Probe, insulating handle, and cap.

Bottom, Probe within insulating handle.

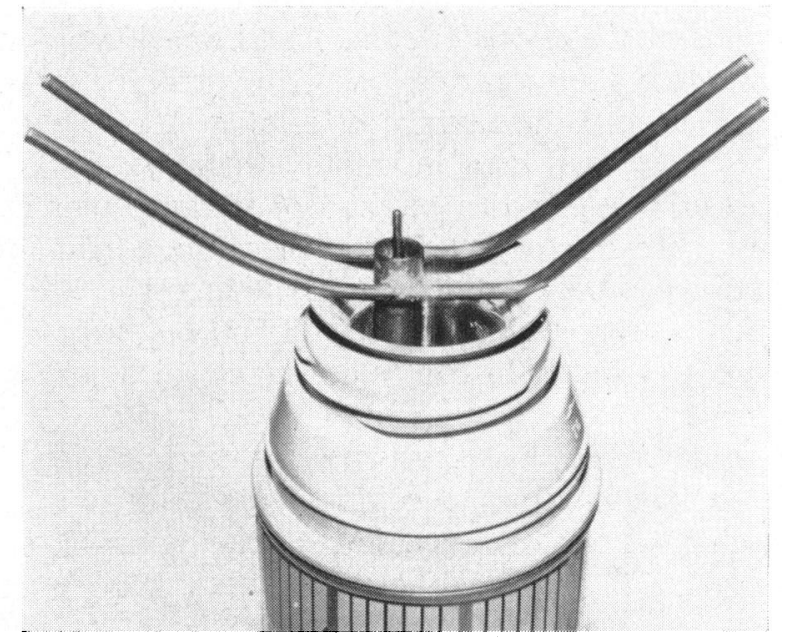

FIG. 2.-Cooling the probe in a vacuum flask.

and non-toxic. Its use as a refrigerant in ophthalmic surgery has been reported by Kelman and Cooper (1963) and Bellows (1964).

\section{Method}

The operations were performed under local anaesthesia with the usual procedures of akinesia, retrobulbar anaesthesia, superior rectus suture, Graefe-knife incision of $180^{\circ}$, and 1 to 3 corneoscleral pure silk sutures. Either a complete or peripheral iridectomy was made, depending on the pupillary dilatation. The former gives better access to the upper pole of the lens and renders unnecessary any retraction of the iris by the assistant. 
The cold probe is removed from the tube in the vacuum flask with forceps and is inserted into a sterilized insulating handle of expanded polyurethane, which leaves $2 \mathrm{~mm}$. of the probe tip exposed. The probe within its insulating handle can then be held comfortably in the hand like a pen. Pressure with a lens hook over the lower limbus causes initial rupture of the lower zonular fibres and tilts the upper pole of the lens forwards. The assistant holds the cornea forwards by tension on the corneoscleral suture; the tip of the cold probe touches the upper pole of the lens. There is immediate local freezing with the appearance of a sharply demarcated white circle of frozen capsule and lens (Fig. 3). After a few seconds the lens becomes firmly attached to the probe. The lens is easily removed by a sliding technique with a combination of pressure from below and rotatory movements above, or following enzymatic zonulolysis by traction alone (Fig. 4).

The tip of the cold probe is dipped momentarily into sterile water immediately before use to obtain a thin coating of ice, which is necessary before the probe will adhere to the lens.

The average age of the patients was 72 years in an age range of 55 to 89 years.

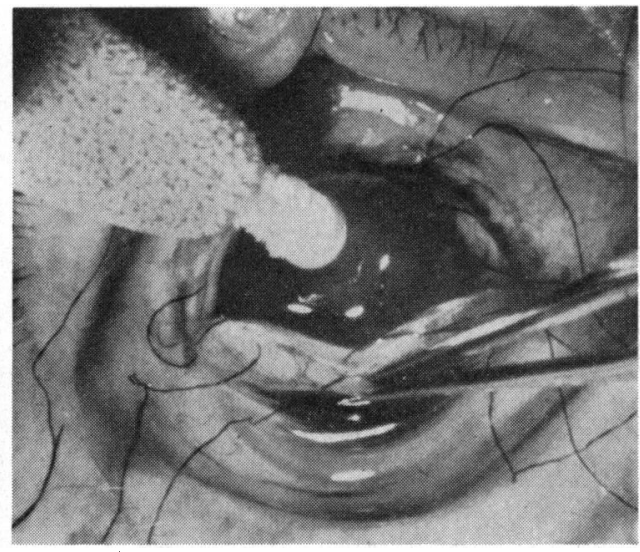

Fig. 3.-Cold probe on the lens, showing white circle of frozen capsule and lens.

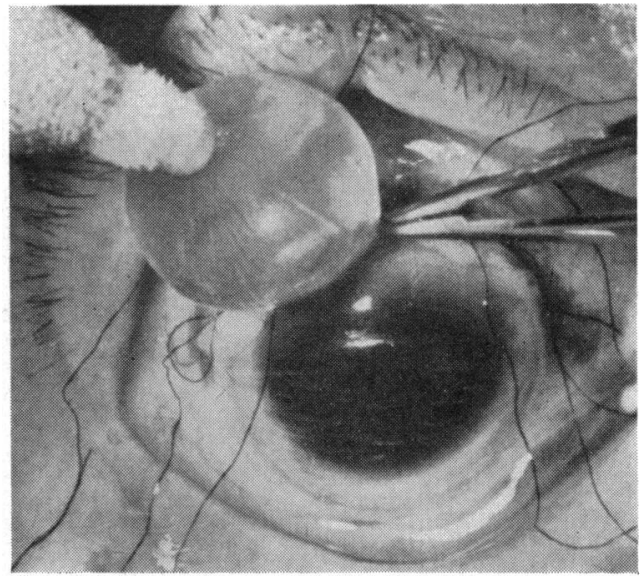

FIG. 4.-Lens removed by traction.

\section{Discussion}

The marked adhesion between the cold probe and lens facilitated intracapsular extraction. The advantage of the method is that it avoids the necessity of grasping the capsule with forceps, a procedure which is often difficult to see and execute.

Traction is transmitted over the wider area of a solid globule of frozen capsule and lens, and not to the capsule alone as in a normal intracapsular extraction. There should therefore be less chance of capsular rupture. In this small series there was one ruptured capsule which was probably due to premature traction on the capsule, before freezing had progressed into the cortex.

Experiments with cadaver lenses and cataracts following extraction have shown that the strength of the ice-bond between the cold probe and lens is several times greater than the strength of the capsule when held in intracapsular forceps.

Kelman and Cooper (1963) found that when the capsule was gripped in forceps it ruptured when supporting a weight of $7.5 \mathrm{~g}$., compared with $250 \mathrm{~g}$. with the lens attached to the cryosurgical applicator at $-20^{\circ} \mathrm{C}$. 
There were no cases of vitreous loss and no evidence of delayed wound healing.

The post-operative appearance of the eyes was no different from that of a normal intracapsular extraction. A chymotrypsin saline 1 in 5,000 solution was used in 5 cases with resultant easy delivery.

In two cases with intumescent cataracts the capsules were taut and smooth and could not be grasped with Castroviejo forceps. These lenses were removed easily with the cold probe.

Because of a fault in technique one of the early cases gave rise to anxiety. The cold probe was placed on the lens capsule and a drop of water was dropped on to the tip of the probe. There was rapid freezing of the water in the anterior chamber and the cornea and iris became firmly attached to the probe. With an iris repositor the cornea was detached, but a large iridectomy was necessary in the sector extending from 9.30 to 1 o'clock. The first dressing showed an almost complete hyphaema, which absorbed in 22 days. Apart from a localized peripheral corneal endothelial opacity between 9.30 and 10 o'clock (which may have been due to the forcible separation of frozen cornea from the probe) there was no evidence of corneal damage. The vision improved to 6/9 despite some macular degeneration. In two other cases the corneal endothelium was touched with the tip of the cold probe. The attachment was promptly separated. Localized corneal cloudiness and folds in Descemet's membrane were present but cleared in 14 days.

Bellows (1964) found that the application of his cryogenic instrument to the corneal endothelium caused corneal cloudiness and cracks and folds in Descemet's membrane. The cornea resumed its normal appearance after four weeks.

Krwawicz (1963) observed no harmful sequelae from this complication. It would appear that momentary localized freezing does not permanently damage the cornea.

If the probe is used immediately after removal from the liquid nitrogen it causes freezing of the lens, but there is little or no adhesion between the probe and lens. After two to three minutes, during which time the temperature of the probe has risen, the adhesive power is much greater. This suggests that lower temperatures and quicker freezing are less effective. Bellows (1964) found that when using liquid nitrogen the ice crystals formed were too small to cause adhesion between the lens and metal tip, and he obtained better adhesion using solid carbon dioxide.

A cryoextractor (Fig. 5), which may contain either solid carbon dioxide (boiling point $-78 \cdot 2{ }^{\circ} \mathrm{C}$.) or liquid nitrogen within the handle, is at present in use experimentally on enucleated and cadaver eyes.

The optimum temperature and rate of freezing most favourable

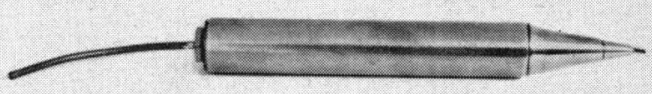

FIG. 5.-Cryoextractor containing solid carbon dioxide or liquid nitrogen.

for cataract extraction have yet to be determined. An instrument is being developed which has improved insulation of the probe tip and thermocouple monitoring. The temperature of the probe is always under the surgeon's control, and can be raised or lowered at will. 


\section{Summary}

Extreme cold is a new surgical tool in cataract surgery. The limited experience gained from this small series of 30 senile cataract extractions suggests that with this technique the difficulties of the intracapsular cataract operation are lessened.

There is no necessity to grasp the capsule and fewer capsules are ruptured. The method is most suitable for extraction of intumescent or hypermature cataracts where the capsule cannot be grasped with forceps.

The hazards of adhesion of the cold probe to cornea, iris, or corneoscleral sutures should be overcome with an instrument now being developed.

I wish to thank Dr I. K. Smith of the Department of Civil and Mechanical Engineering (Thermodynamics Section), Northampton College of Advanced Technology, London; Dr. G. T. Davies, until recently Ophthalmic Registrar, Whipps Cross Hospital, London, for his care of the patients; and Mr. F. A. D. Allen, Group Pharmacist at this hospital for his assistance.

\section{REFERENCES}

Bellows, J. G. (1964). Amer. J. Ophthal., 57, 29.

Billingham, R. E. (1957). Proc. roy. Soc. B, 147, 530.

Davies, G. T. (1965). Brit. J. Ophthal., 49, 155.

Kelman, C. D., and COOPER, I. S. (1963). Amer. J. Ophthal., 56, 731.

KrWAwICZ, T. (1961). Brit. J. Ophthal., 45, 279. (1963). Ibid., 47, 36.

RIDGe, J. W. (1957). Proc. roy. Soc. B, 147, 531. 